\title{
Precise Microacoustic Characterization of new Piezoelectric Crystals for High-Temperature Sensors
}

\author{
H. Schmidt ${ }^{1}$, A. Sotnikov ${ }^{1,2}$, S.V. Biryukov ${ }^{1}, M$ Weihnacht $^{1}$ \\ 1 IFW Dresden, SAWLab Saxony, Helmholtzstr. 20, 01069 Dresden, Germany, \\ h.schmidt@ifw-dresden.de \\ ${ }^{2}$ A.F. Ioffe Physical-Technical Institute, Polytekhnicheskaya 26, 194021 St. Petersburg, Russia
}

\begin{abstract}
:
Promising new single crystals for piezoelectric sensors with high-temperature capability were precisely characterized by a powerful combination of bulk acoustic wave (BAW) and surface acoustic wave (SAW) measurements. Crystals from the langasite $\left(\mathrm{Ca}_{3} \mathrm{TaGa}_{3} \mathrm{Si}_{2} \mathrm{O}_{14}\right)$ and the oxoborate $\left(\mathrm{GdCa} 4 \mathrm{O}\left(\mathrm{BO}_{3}\right)_{3}\right.$ and $\left.\mathrm{LaCa}_{4} \mathrm{O}\left(\mathrm{BO}_{3}\right)_{3}\right)$ family have been investigated in detail and material data were derived with high accuracy at room temperature. Additionally, their behavior was investigated up to high temperatures of about $1000^{\circ} \mathrm{C}$ demonstrating the capability for high-temperature piezoelectric sensors.
\end{abstract}

Key words: piezoelectric crystal, bulk acoustic wave, surface acoustic waves, langasite, oxoborate, high-temperature sensors

\section{Introduction}

Piezoelectric single crystals are widely used as transducers, bulk acoustic wave (BAW) and surface acoustic wave (SAW) filters and resonators, actuators and especially as sensors for various physical quantities. Such devices require single crystals with sufficiently high electromechanical coupling efficiencies and low dielectric as well as acoustic loss in a wide temperature range, e.g. from room temperature up to very high temperatures of about $1000^{\circ} \mathrm{C}$. Besides, the precise knowledge of the acoustic material constants, comprising the elastic, piezoelectric, and dielectric tensor elements and their temperature dependencies is an indispensable pre-condition for the successful device design and an appropriate selection of applications. Over the last years, many efforts have been done to develop new piezoelectric crystals with improved physical parameters and high thermal stability in a wide temperature range. Among them are the members of the langasite family [1-6] which belong to the same trigonal crystal class 32 like the well-known quartz and the oxoborate family [6-10] (monoclinic crystal class $\mathrm{m}$ ). The crystals from both families demonstrate a combination of relatively high values of piezoelectric coupling coefficients, small acoustic loss and high operating temperature limits due to the absence of a phase transition below their melting point. The other important feature of langasites and oxoborates is the availability of large size boules of good quality grown by the welldeveloped and relatively inexpensive Czochralski technique.

For a comprehensive microacoustic characterization, one needs a special strategy for the precise determination of complete sets of material parameters. To our mind, the best way to overcome the emerging problem of different accuracy of certain parameters seems to be the combination of different methods of specific appropriateness for each type of material constant. In the case of elastic coefficients, we used the pulse-echo ultrasonic technique for the determination of BAW phase velocities in a wide temperature range, partially supplemented by laser-acoustic measurements of SAW phase velocity at room temperature. Both methods were performed for crystal cuts of different crystallographic orientations and propagation directions as well as with different wave polarizations allowing to extract almost all material constants.

\section{Crystal growth and samples preparation}

$\mathrm{Ca}_{3} \mathrm{TaGa}_{3} \mathrm{Si}_{2} \mathrm{O}_{14}$ (CTGS) single crystals were grown by the Czochralski technique in an inductively heated iridium crucible by FOMOS Materials, Moscow, Russia [11]. Gadolinium oxoborate $\mathrm{GdCOB}\left(\mathrm{GdCa} 4 \mathrm{O}\left(\mathrm{BO}_{3}\right)_{3}\right)$ and Lanthanum oxoborate $\mathrm{LaCOB}\left(\mathrm{LaCa} 4 \mathrm{O}\left(\mathrm{BO}_{3}\right)_{3}\right)$ single crystals were grown at TU Bergakademie Freiberg, Germany using the Czochralski 
technique as well. For the ultrasonic wave velocity measurements of CTGS cube-like samples of approximately $7 \times 7 \times 7 \mathrm{~mm}^{3}$ were cut from the boule in three different orientations: (a) with the faces perpendicular to the main $X$, $Y, Z$ axes ([100], [010] and [001] directions, respectively), (b) rotated by $+45^{\circ}$ around the $X$ axis and (c) rotated by $-45^{\circ}$ around the $X$ axis. For the determination of the dielectric constants thin plates of $\mathrm{X}$-cut and Z-cut with averaged dimensions of $10 \times 10 \times 0.5 \mathrm{~mm}^{3}$ were used. Laser-acoustic SAW phase velocity measurements were carried out on wafer samples of different sizes (diameter: 1.5" and 3") with a thickness of ca. $0.53 \mathrm{~mm}$. Here, also cuts of different orientation were investigated: $\mathrm{X}$ - and $\mathrm{Y}$-cut as well as the rotated cuts: $\mathrm{Y}$ $21.3^{\circ}, Y+45^{\circ}$ and $Y-45^{\circ}$ which means a rotation by the specified angle around the main $X$-axis (i.e. with respect to $Y$-cut). In the case of oxoborates ultrasonic wave velocity measurements were done using crystal cubes of about $8 \times 8 \times 8 \mathrm{~mm}^{3}$ and bars of about $8 \times 5 \times$ $5 \mathrm{~mm}^{3}$ in size cut from the as grown boule such they have faces normal to the [100], [010], [001], and [101] directions. Thin plates of about $8 \times 8 \times 1 \mathrm{~mm}^{3}$ were used for dielectric and electromechanical coupling coefficient measurements. Orientation accuracy was within $0.1^{\circ}$ for all samples. Surfaces of wafers used for SAW velocity measurements were finally coated with a $20 \mathrm{~nm}$ thin $\mathrm{Al}$ layer to enhance the laser-acoustic SAW excitation.

\section{Experimental procedure}

Measurements of the bulk acoustic wave velocities propagating along certain crystallographic directions were carried out by means of a RITEC Advanced Ultrasonic Measurement System RAM-5000. Our setup realizes the pulse-echo method of time propagation measurements and supports operation at 5,10 and $30 \mathrm{MHz}$ in the sample temperature range $25^{\circ} \mathrm{C}-995^{\circ} \mathrm{C}$ with an accuracy of about $10^{-4}$. All temperature dependent measurements were performed using a Carbolite tube furnace with a working temperature range up to $1200^{\circ} \mathrm{C}$ at a proven temperatur accuracy and stability of $0.5^{\circ} \mathrm{C}$.

The SAW phase velocity measurements were carried out using laser-based thermo-elastic SAW generation and piezoelectric probing without use of interdigital transducers. In general the system allows to operate samples in the restricted temperature range $-20^{\circ} \mathrm{C} . . .70^{\circ} \mathrm{C}$ for frequencies up to $>300 \mathrm{MHz}$ and exhibits an accuracy of $10^{-4}$ depending on sample size and quality. At room temperature even a better accuracy may be obtained. The experimental setup is shown schematically in Fig. 1.
Broadband SAW pulses are excited by a nitrogen pulse laser $(\lambda=337.1 \mathrm{~nm}, \mathrm{~T}=0.5 \mathrm{~ns})$ which is focused onto the sample surface by a cylindrical lens. The fast expansioncompression-pulse of the heated focus area yields elastic SAW bursts propagating along the surface. These pulses are finally transformed into electric signal by a broadband transducer comprising a piezoelectric PVDF foil pressed onto the sample surface by a steel wedge. The pulses are then amplified and processed by a digital oscilloscope. For the SAW phase velocity measurement the sample is moved by a high precision translation stage (accuracy: 25nm) with respect to the laser beam position and SAW pulses are received at different well defined distances from the origin.

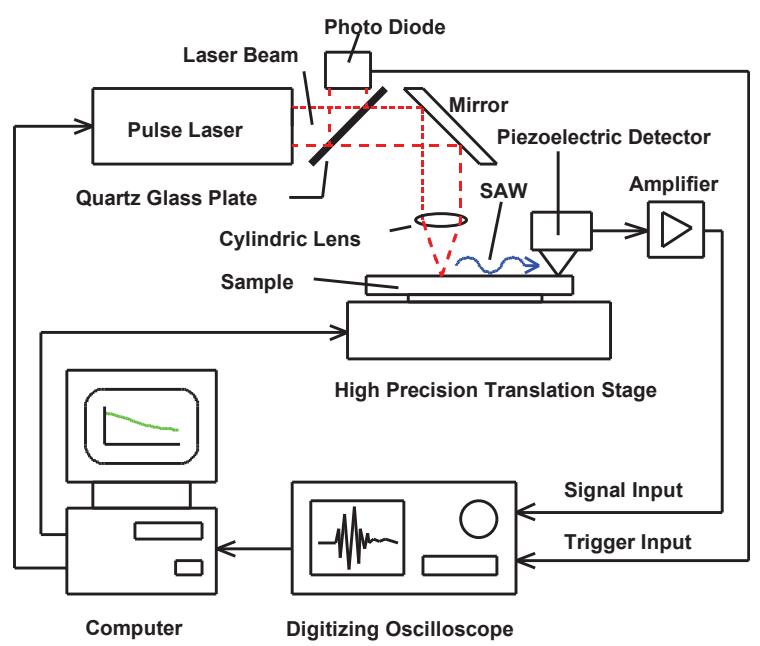

Fig. 1. Laser-pulse setup for SAW phase velocity measurements.

Beside BAW and SAW experiments usual resonance technique and dielectric measurements have been used for determining electromechanical coupling and dielectric constants. The Archimedes method was used to measure the mass densities. The room temperature values were found to be 4.62 $\mathrm{g} / \mathrm{cm}^{3}, 3.70 \mathrm{~g} / \mathrm{cm}^{3}$ and $3.49 \mathrm{~g} / \mathrm{cm}^{3}$ for CTGS, GdCOB and LaCOB, respectively, what is in a good accordance with the published data.

\section{Results and discussion}

The elastic coefficients $C_{i j}$ and piezoelectric constants $e_{i j}$ for CTGS can be derived using a system of relations between bulk sound velocities measured at different directions for different propagation modes assuming the mass density $\rho$ and components of the dielectric constant tensor $\varepsilon_{i j}$ are known (see for example [3]). Using material parameters obtained from BAW [12] and from laser-pulse SAW measurements [15] in a variety of propagation directions on wafers of different crystallographic orientations (i.e. cuts) an 
improved set of CTGS material parameters at room temperature has been derived (see Tab. 1). Calculated results of related angular dispersions of SAW phase velocity are shown in Fig. 2. For comparison, curves based on BAW data from [13] and [14] are also included.

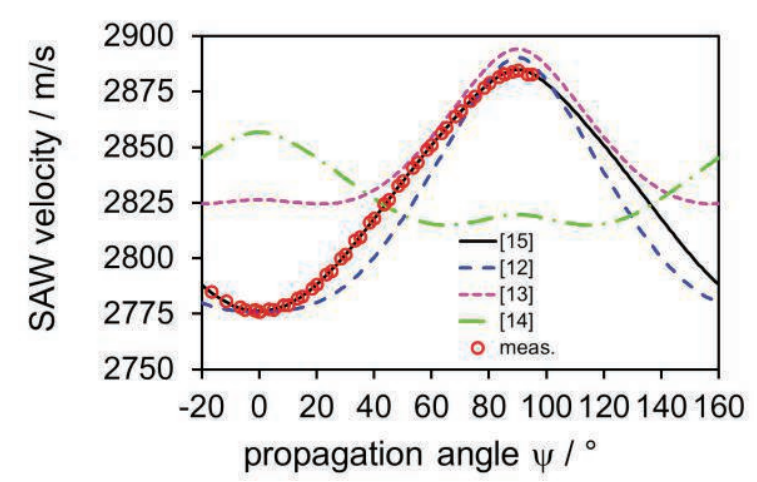

Fig. 2. Calculated angular SAW velocity dispersion curves for Y-cut CTGS (Euler angles $\left(0^{\circ},-90^{\circ}, \psi\right)$ based on different material data sets compared to laser-acoustic SAW measurements.

SAW velocities as a function of propagation angle for $X$ - and rotated $Y \pm 45^{\circ}$-cuts using the improved set of material constants are shown in Figs. 3-5.

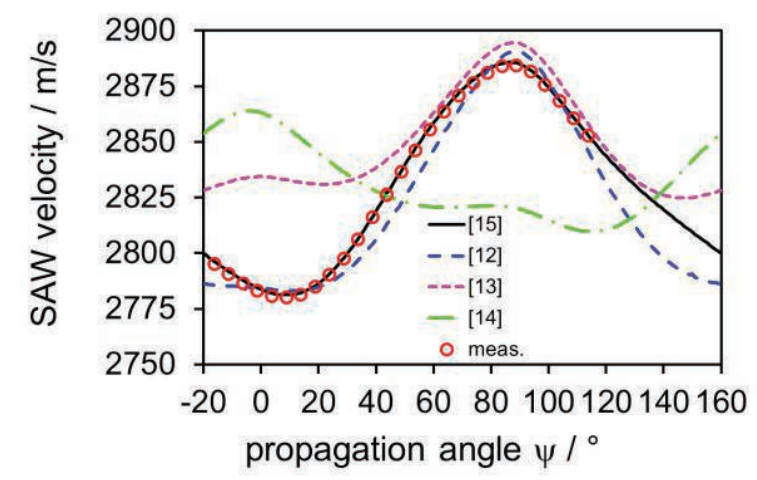

Fig. 3. Calculated angular SAW velocity dispersion as a function of propagation angle for X-cut CTGS (Euler angles $\left(90^{\circ}, 90^{\circ}, \psi\right)$ based on different data sets compared to measured SAW velocity data.

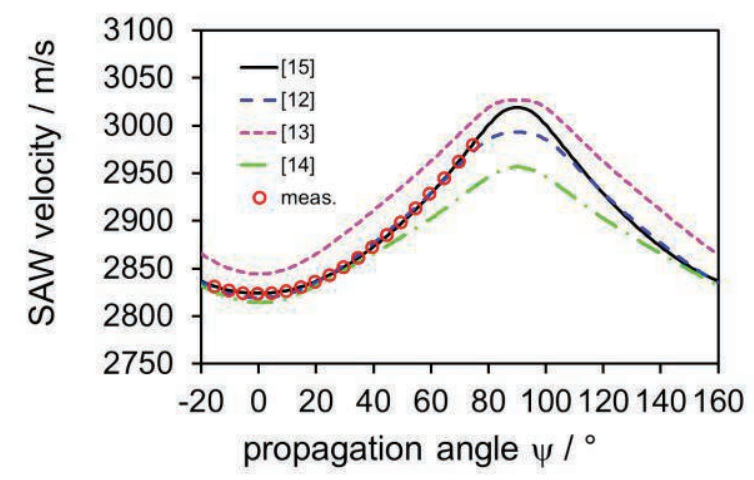

Fig. 4. Calculated angular SAW velocity dispersion as a function of propagation angle for $\mathrm{Y}+45^{\circ}$-cut CTGS (Euler angles $\left(0^{\circ},-45^{\circ}, \psi\right)$ based on different data sets compared to measured SAW velocity data.

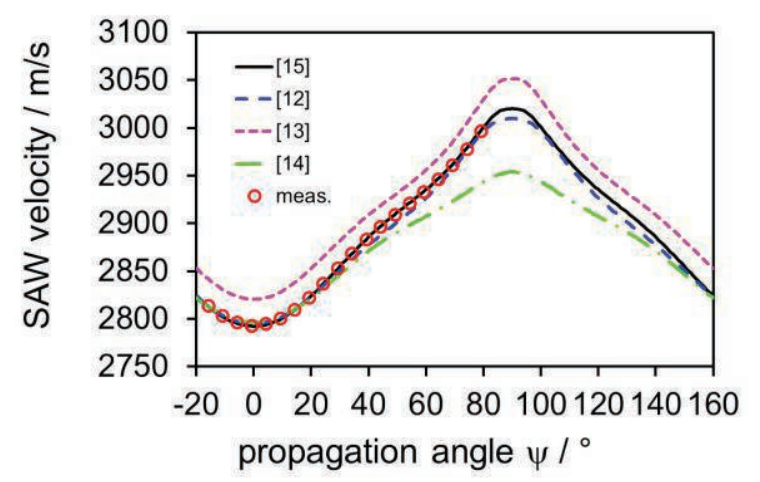

Fig. 5. Calculated angular SAW velocity dispersion as a function of propagation angle for $\mathrm{Y}-45^{\circ}$-cut CTGS (Euler angles $\left(0^{\circ},+45^{\circ}, \psi\right)$ based on different data sets compared to measured SAW velocity data.

Notice the excellent coincidence of laseracoustic measurement results and simulations based on the improved data set [15] for all cuts and the whole range of propagation directions.

Tab. 1 summarizes the sets of material parameters obtained using BAW only and simultaneous SAW measurements and calculations. Comparing the set of constants finally extracted from SAW measurements with those obtained from BAW measurements only we can conclude a good general agreement for almost all constants with the exception of elastic constant $\mathrm{C}_{13}$ and piezoelectric coefficient $\mathrm{e}_{14 .}$.

Tab. 1: Material parameters of CTGS single crystal (rounded values after [12] (based on BAW) and after [15] (BAW, refined by SAW), resp.)

\begin{tabular}{|c|c|c|}
\hline $\begin{array}{l}\text { Material } \\
\text { constant }\end{array}$ & BAW data & $\begin{array}{c}\text { BAW \& SAW } \\
\text { data }\end{array}$ \\
\hline $\mathrm{C}_{11} / \mathrm{GPa}$ & 157.4 & 154.8 \\
\hline $\mathrm{C}_{12} / \mathrm{GPa}$ & 75.8 & 73.0 \\
\hline $\mathrm{C}_{13} / \mathrm{GPa}$ & 64.2 & 70.5 \\
\hline $\mathrm{C}_{14} / \mathrm{GPa}$ & 0.537 & 0.635 \\
\hline $\mathrm{C}_{33} / \mathrm{GPa}$ & 210.6 & 211.3 \\
\hline $\mathrm{C}_{44} / \mathrm{GPa}$ & 41.8 & 42.0 \\
\hline $\mathrm{C}_{66} / \mathrm{GPa}$ & 40.8 & 40.9 \\
\hline $\mathrm{e}_{11} / \mathrm{C} / \mathrm{m}^{2}$ & -0.405 & -0.378 \\
\hline $\mathrm{e}_{14} / \mathrm{C} / \mathrm{m}^{2}$ & 0.283 & 0.631 \\
\hline$\varepsilon_{11} / \varepsilon_{0}$ & 18.2 & 18.2 \\
\hline$\varepsilon_{13} / \varepsilon_{0}$ & 23.9 & 23.9 \\
\hline$\rho / \mathrm{kg} / \mathrm{m}^{3}$ & 4620 & 4620 \\
\hline
\end{tabular}

For the oxoborate crystals several material data have been derived so far from measurements on bulk samples only. Temperature 
dependences of the dielectric constant $\varepsilon_{11}$ for GdCOB and LaCOB are shown in Fig. 6. Notice that the most important temperature behavior is that of $\varepsilon_{11}$ and $\varepsilon_{22}$ since [100] and [010] crystal cuts show reasonably high electromechanical coupling coefficients for shear ultrasonic modes. It is seen that dielectric constants slightly increase with increasing temperature. Such a behavior is typical for ordinary linear dielectrics. As mentioned above, low dielectric loss at high temperature is an important mandatory feature for piezoelectric crystal candidates to be selected for high temperature sensor applications.

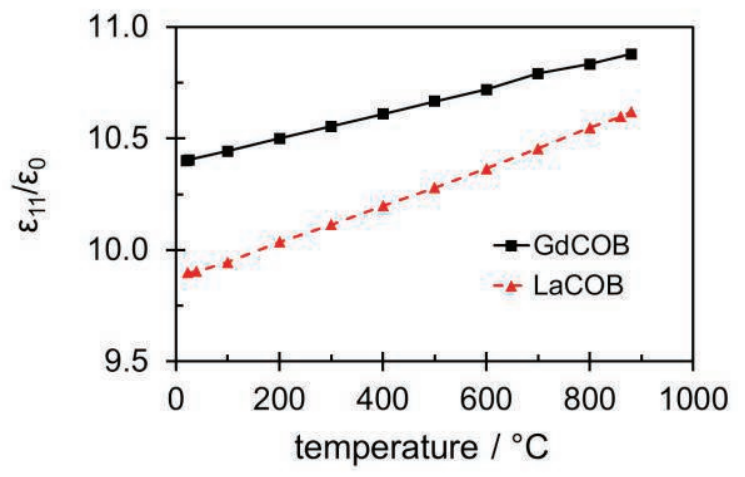

Fig. 6. Dielectric constant $\varepsilon_{11} / \varepsilon_{0}$ as a function of temperature for oxoborates (GdCOB, LaCOB).

Dielectric loss curves versus temperature are depicted in Fig. 7. It can be seen, that a relatively low loss at high temperatures is found for GdCOB while LaCOB exhibits relatively high loss at $\mathrm{T}>700^{\circ} \mathrm{C}$. In Fig. 7 also included for comparison are our results for the well-known langasite (LGS) single crystal. It is obvious that at high temperatures both oxoborates crystals show lower dielectric loss as compared to the commonly used langasite.

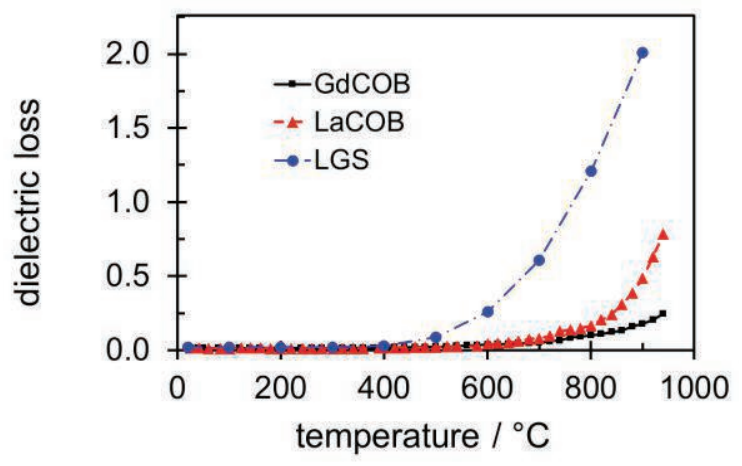

Fig. 7. Dielectric loss as a function of temperature for oxoborates (GdCOB, LaCOB) and langasite (LGS).

Also, electromechanical coupling is a very important characteristic for the application of piezoelectric crystals in sensors. As it follows from our results, the most interesting directions in GdCOB and LaCOB are with [010] and [100] cuts for exciting shear waves using the internal piezoelectric effect of the crystal. Here the resonant frequencies of the fundamental and first two odd harmonic overtones of the thickness modes of thin plates (bulk resonators) were measured. The ratio of the fundamental and odd overtone frequencies yields the corresponding coefficient of electromechanical coupling by the overtone ratio method [16]. As an example the electromechanical coupling coefficients versus temperature for [010] plates are shown in Fig. 8.

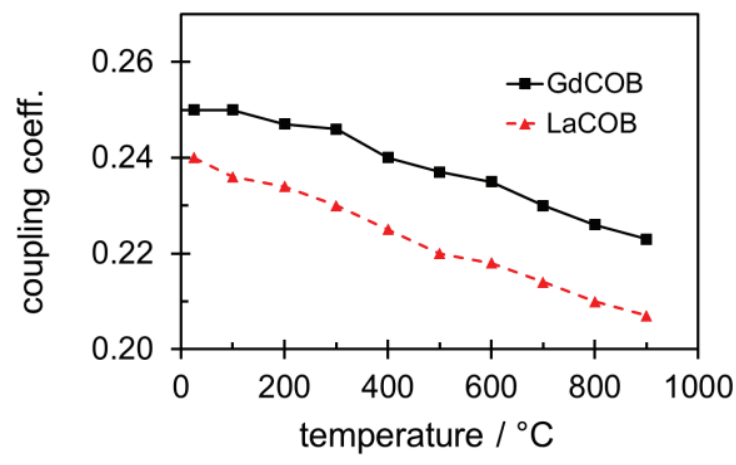

Fig. 8. Electromechanical coupling coefficients as a function of temperature for oxoborate crystals.

As to be seen the electromechanical coupling coefficients were found to decrease a little with increasing temperature, nevertheless they remain large enough at the highest temperature during the experiments.

To demonstrate the strong piezoelectric activity in the oxoborate samples even at very high temperatures we used the direct excitation of the acoustic waves by the internal piezoelectric effect of the crystal. Fig. 9 shows as an example the ultrasonic pulse-echo signal pattern at $10 \mathrm{MHz}$ for the piezoelectrically active shear mode propagating along [010] direction in $\mathrm{GdCOB}$ at $995^{\circ} \mathrm{C}$.

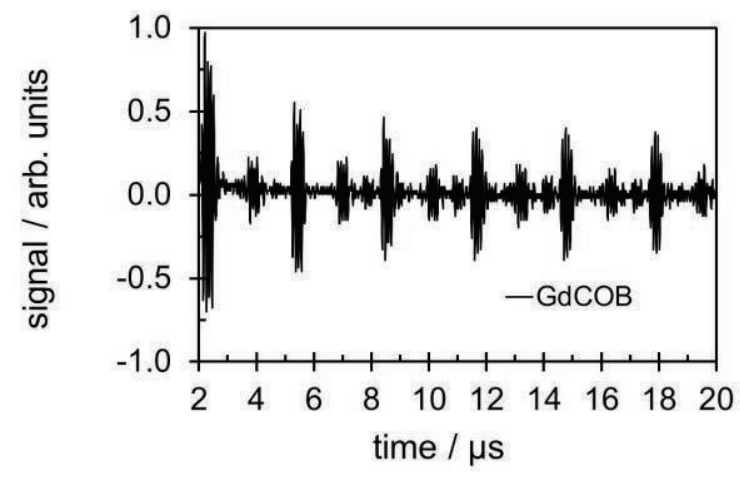

Fig. 9. Pulse-echo pattern (frequency $10 \mathrm{MHz}$ ) for the shear mode propagating along [010] direction in $\mathrm{GdCOB}$ single crystal at $995^{\circ} \mathrm{C}$. 
Worth mentioning that for a monoclinic crystal, the extraction of material constants from ultrasonic velocity data is generally a challenging task. Here, to our mind precise measurement of the sound velocities of bulk samples using ultrasonic pulse-echo technique provides the most reliable results as compared to other experimental methods. To obtain all elastic constants, ultrasonic velocities for 18 different modes propagating along six directions are essential. Therefore, cumbersome experimental and computational procedures are required. In course of the present work, longitudinal and shear velocities propagating along 6 directions have been measured. As a result, 10 from 13 elastic constants have been obtained. The results are presented in Tab. 2.

Tab. 2: Elastic constants of GdCOB and LaCOB crystals at room temperature

\begin{tabular}{|c|c|c|}
\hline $\begin{array}{c}\text { constant } \\
/ \mathrm{GPa}\end{array}$ & GdCOB & LaCOB \\
\hline $\mathrm{C}_{11}$ & 160.5 & 169.1 \\
\hline $\mathrm{C}_{13}$ & 85.3 & 73.02 \\
\hline $\mathrm{C}_{15}$ & 2.8 & 3.07 \\
\hline $\mathrm{C}_{22}$ & 163.5 & 165.5 \\
\hline $\mathrm{C}_{33}$ & 154.0 & 149.3 \\
\hline $\mathrm{C}_{35}$ & 5.0 & 7.9 \\
\hline $\mathrm{C}_{44}$ & 24.8 & 26.6 \\
\hline $\mathrm{C}_{46}$ & -9.07 & -1.2 \\
\hline $\mathrm{C}_{55}$ & 39.5 & 42.7 \\
\hline $\mathrm{C}_{66}$ & 54.37 & 53.3 \\
\hline
\end{tabular}

To demonstrate the temperature capabilities of the oxoborates under investigation figs. 10-11 show as an example the temperature dependences of elastic constants important for possible applications of $\mathrm{GdCOB}$ and $\mathrm{LaCOB}$ crystals.

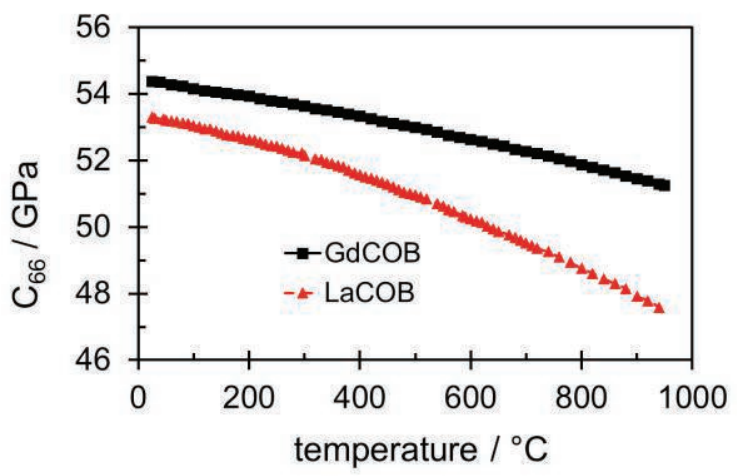

Fig. 10. Elastic constant $C_{66}$ as a function of temperature for GdCOB and LaCOB single crystal.

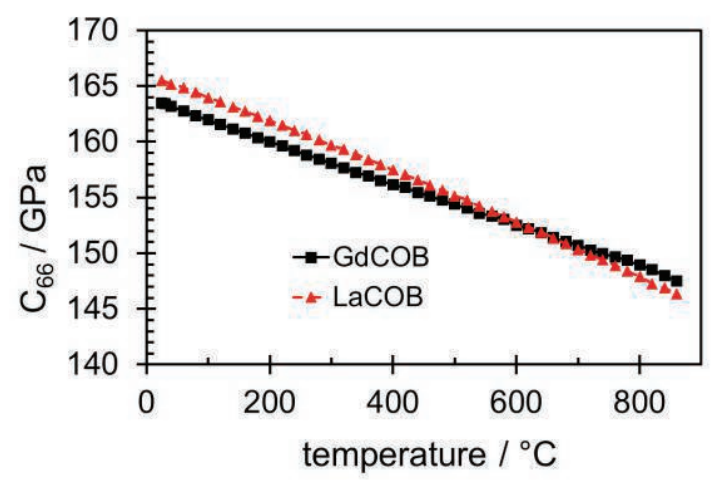

Fig. 11. Elastic constant $C_{22}$ as a function of temperature for GdCOB and LaCOB single crystal.

As expected the elastic constants corresponding to both shear and longitudinal modes show the usual temperature change: a monotonic decrease with increasing temperature.

\section{Conclusion}

Using the pulse-echo ultrasonic method (BAW) and the laser-acoustic SAW technique supplemented by appropriate parameter extraction calculations promising high temperature capable piezoelectric crystals from langasite (CTGS) and oxoborate (GdCOB and LaCOB) families have been investigated. A optimized set of CTGS material parameters has been derived. Elastic constants important for acoustic applications were investigated for GdCOB and LaCOB oxoborates from room temperature up to the high temperature region around $900^{\circ} \mathrm{C}-950^{\circ} \mathrm{C}$. Additionally the electromechanical coupling coefficients as well as dielectric properties were measured in a wide temperature range. A strong piezoelectric excitation of shear waves propagating along [100] and [010] directions was maintained by the investigated oxoborates up to $995^{\circ} \mathrm{C}$.

\section{Acknowledgements}

This work was supported by DFG grant (SO 1085/1-1) and BMBF grant (InnoProfileTransfer 03IPT610Y). The authors would like to express their gratitude to Prof. J. Götze and M. Hengst (Freiberg University of Mining and Technology), Drs. S. Sakharov and O. Buzanov (FOMOS Materials) for high quality crystals.

\section{References}

[1] S. Sakharov, P. Senushencov, A. Medvedev, Yu. Pisarevsky, New data on temperature stability and acoustical losses of langasite crystals, Proc. IEEE Int. Freq. Contr. Symp., 647-652 (1995).

[2] J. Bohm, E. Chilla, C. Flannery, H.-J. Fröhlich, T. Hauke, R.B Heimann, M. Hengst, U. Straube, Czochralski growth and characterization of piezoelectric single crystals with langasite 
structure: $\mathrm{La}_{3} \mathrm{Ga}_{5} \mathrm{SiO}_{14}$ (LGS), $\mathrm{La}_{3} \mathrm{Ga}_{5.5} \mathrm{Nb}_{0.5} \mathrm{O}_{14}$ (LGN) and $\mathrm{La}_{3} \mathrm{Ga}_{5.5} \mathrm{Ta}_{0.5} \mathrm{O}_{14}$ (LGT). II.

Piezoelectric and elastic properties, J. Cryst. Growth 216, 293-298 (2000).

[3] J.A. Kosinski, R.A. Pastore, E Bigler, M. Pereira da Cunha, D.C. Malocha, J. Detaint, A review of langasite material constants from BAW and SAW data: toward an improved data set, Proc. IEEE Int. Freq. Contr. Symp., 278-286 (2001).

[4] E. Chilla, R. Kunze, M. Weihnacht, J. Bohm, R.B. Heimann, M. Hengst, U. Straube, Acoustic waves measurements on SNGS crystals and determination of material constants, Proc. IEEE Ultrason. Symp., 92-95 (2003).

[5] A.V. Sotnikov, M. Weihnacht, H. Schmidt, K. Suschke, M. Hengst, J. Götze, Piezoelectric and elastic properties of SNGS and STGS at elevated temperatures, Proc. IEEE Ultrason. Symp., 22252228 (2008).

[6] S. Zhang and F. Yu, Piezoelectric materials for high temperature sensors, J. Am. Ceram. Soc. 94, 3153-3170 (2011).

[7] H. Shimizu, T. Nishida, H. Takeda, T. Shiosaki, Dielectric, elastic and piezoelectric properties of $\mathrm{RCa}{ }_{4} \mathrm{O}\left(\mathrm{BO}_{3}\right)_{3}(\mathrm{R}=$ rare-earth elements) crystals with monoclinic structure of point group $\mathrm{m}, \mathrm{J}$. Cryst. Growth 311, 916-920 (2009).

[8] T. Karaki, M. Adachi, Y. Kuniyoshi, Evaluation of material constants in $\mathrm{NdCa}{ }_{4} \mathrm{O}\left(\mathrm{BO}_{3}\right)_{3}$ piezoelectric single crystal, J. Electroceramics 21, 823-826 (2008).

[9] F.P. Yu, S. Zhang, X. Zhao, D. Yuan, C.-M. Wang, T.R. Shrout, Characterization of neodimium calcium oxyborate piezoelectric crystal with monoclinic phase, Cryst. Growth Design 10, 1871-1877 (2010).

[10] F. Yu, S. Zhang, X. Zhao, D. Yuan, L. Qin, Q.-M. Wang, T.R. Shrout, Dielectric and electromechanical properties of rare earth calcium oxyborate piezoelectric crystals at high temperatures, IEEE Trans. UFFC 58, 868-873 (2011).

[11] D.V. Roshchupkin, D.V. Irzhak, E.V. Emelin, R.R. Fahrtdinov, O.A. Plotitsyna, S.A. Sakharov, O.A. Buzanov, A.N. Zabelin, Advanced piezoelectric crystal $\mathrm{Ca}_{3} \mathrm{TaGa}_{3} \mathrm{Si}_{2} \mathrm{O}_{14}$ : growth, crystal structure perfection, piezoelectric and acoustic properties, Proc. IEEE Ultrason. Symp., 2730-2733 (2012).

[12] A. Sotnikov, H. Schmidt, M. Weihnacht, O. Buzanov, S. Sakharov, Material parameters of $\mathrm{Ca}_{3} \mathrm{TaGa}_{3} \mathrm{Si}_{2} \mathrm{O}_{14}$ single crystal revisited, Proc. IEEE Ultrason. Symp., 1688-1691 (2013).

[13] Yu.V. Pisarevsky, B.V. Mill, N.A. Moiseeva, A.V. Yakimov, Ordered $\mathrm{Ca}_{3} \mathrm{TaGa}_{3} \mathrm{Si}_{2} \mathrm{O}_{14}$ crystals: growth, electromechanical and optical properties, Proc. 18 Eur. Frequency and Time Forum, 216219 (2004).

[14] X. Shi, D. Yuan, X. Yin, A. Wei, S. Guo, F. Yu, Crystal growth and dielectric, piezoelectric and elastic properties of $\mathrm{Ca}_{3} \mathrm{TaGa}_{3} \mathrm{Si}_{2} \mathrm{O}_{14}$ single crystal, Solid State Comm. 142, 173-176 (2007).

[15] S.V. Biryukov, H. Schmidt, A. Sotnikov, M. Weihnacht, S. Sakharov, O. Buzanov, CTGS material parameters obtained by versatile SAW measurements, Proc. IEEE Ultrason. Symp., 882885 (2014).

[16] M. Onoe, H.F Tiersten, A.H. Meitzler, Shift in the location of resonant frequencies caused by large electromechanical coupling in thickness-mode resonators, J. Acoust. Soc. Amer. 35, 36-42 (1963). 